\title{
AVAILABILITY ANALYSIS OF PARALLEL TRANSIT FUEL SYSTEM IN PETROL ENGINE UNDER HEAD OF LINE REPAIR
}

\author{
Reena Garg ${ }^{1}$ \\ ${ }^{1}$ Assistant Professor Mathematics, YMCA University of Science \& Technology, Faridabad 121006
}

\begin{abstract}
In this paper, the author has considered a fuel system in petrol engine with one alternative fuel source (like LPG, CNG etc.) keeping in standby arrangement and followed online through a perfect switching device. The whole system can fail due to either its normal working or human error. All the failures follow exponential time distribution where as all repairs follow general time distribution.
\end{abstract}

Keywords: Fuel system, standby, exponential time distribution, general time distribution.

\section{INTRODUCTION}

In this paper, the considered fuel system consists of three subsystems, namely $A, B$ and $C$, connected in series. Subsystem $A$ is fuel source having two units in standby redundancy. Subsystem $B$ consists of two units (Transfer pump and carburetor) connected in series while subsystem $C$ having $\mathrm{N}$-cylinders [identical] in series and is of $\mathrm{j}$-out-of$\mathrm{N}$ :G nature, fig-1 shows the system configuration. Head-ofline policy is being adopted for repair purpose. The concept of human-error is also incorporated to make the model more compatible. Laplace transform of various state probabilities, availability and long run availability, some particular cases and profit function for the system are obtained. Fig-2 shows the state-transition diagram and states descriptions have mentioned in table- 1 .

By using supplementary variables technique and Laplace transform, various reliability parameters have been obtained. One numerical example together with graphical illustration has also been given at the end to highlight important results of the study.

Table-1: States Description

\begin{tabular}{|c|c|c|c|c|c|}
\hline \multirow[t]{2}{*}{ STATE } & \multicolumn{3}{|c|}{ SUBSYSTEM } & \multirow{2}{*}{$\begin{array}{l}\text { SYSTEM'S } \\
\text { STATE }\end{array}$} & \multirow{2}{*}{$\begin{array}{l}\text { SUPPLEMENTARY } \\
\text { VARIABLE USED }\end{array}$} \\
\hline & $\mathbf{A}$ & B & $\mathbf{C}$ & & \\
\hline $\mathrm{S}_{1}$ & 0 & 0 & 0 & 0 & Nil \\
\hline $\mathrm{S}_{2}$ & 0 & $\bar{F}$ & 0 & $\mathrm{~F}$ & $\mathrm{u}$ \\
\hline $\mathrm{S}_{3}$ & 0 & 0 & $(\mathrm{~N}-\mathrm{j}) 0$ & 0 & Nil \\
\hline $\mathrm{S}_{4}$ & 0 & 0 & $\mathrm{~F}$ & $\mathrm{~F}$ & $\mathrm{v}$ \\
\hline $\mathrm{S}_{5}$ & $1 \mathrm{~F}$ & 0 & $(\mathrm{~N}-\mathrm{j}) 0$ & 0 & Nil \\
\hline $\mathrm{S}_{6}$ & $1 \mathrm{~F}$ & $\mathrm{~F}$ & $(\mathrm{~N}-\mathrm{j}) 0$ & $\mathrm{~F}$ & $\mathrm{y}$ \\
\hline $\mathrm{S}_{7}$ & 0 & $\mathrm{~F}$ & $(\mathrm{~N}-\mathrm{j}) 0$ & $\mathrm{~F}$ & $\mathrm{u}$ \\
\hline $\mathrm{S}_{8}$ & $1 \mathrm{~F}$ & 0 & 0 & 0 & Nil \\
\hline $\mathrm{S}_{9}$ & $1 \mathrm{~F}$ & $\mathrm{~F}$ & 0 & $\mathrm{~F}$ & $\mathrm{y}$ \\
\hline $\mathrm{S}_{10}$ & $\mathrm{FH}$ & $\mathrm{FH}$ & $\mathrm{FH}$ & $\mathrm{FH}$ & $\mathrm{m}$ \\
\hline $\mathrm{S}_{11}$ & $\mathrm{~F}$ & 0 & 0 & $\mathrm{~F}$ & $\mathrm{n}$ \\
\hline $\mathrm{S}_{12}$ & $\mathrm{~F}$ & 0 & $(\mathrm{~N}-\mathrm{j}) 0$ & $\mathrm{~F}$ & $\mathrm{r}$ \\
\hline
\end{tabular}

O: All Operable; F : all Failed; FH : failure due to human-error; $\mathrm{rF}$ :r units have failed; $\mathrm{rO}: \mathrm{r}$ units are operable.

\section{ASSUMPTIONS}

The following assumptions have been associated with this model:

1. Initially, all the units are new and the system is in good condition of full efficiency.

2. Repair has given only when the system is in failed condition and a single service channel is available.
3. After repair, system works like a new one and never damages anything.

4. 1-A unit is a petrol tank while 2-A unit is an alternative fuel source (like CNG, LPG etc.) and the perfect switching device has been used to online the standby unit. 


\section{NOTATIONS}

The following notations have been used throughout the model:

$P_{K}(t)$

: The probability that at time $t$, the system is operable and is in $\mathrm{K}^{\text {th }}$ state, where $\mathrm{K}=1,3,5,8$.

$P_{K}(s, t) \Delta:$ The probability that at time $t$, the system is in $r t h$ failed state and elapsed repair time lies in the interval $(s, s+\Delta)$. $\mathrm{K}=2,4,6,7,9,10,11,12$ and $\mathrm{s}=\mathrm{u}, \mathrm{v}, \mathrm{y}$, $\mathrm{u}, \mathrm{y}, \mathrm{m}, \mathrm{n}, \mathrm{r}$ respectively.

$b_{1} / b_{2} \quad:$ Constant failure rate of $1 \mathrm{~B} / 2 \mathrm{~B}$ unit and note that $b_{1}+b_{2}=b$ (say).

$\mathrm{a} / \mathrm{c} \quad$ : Constant failure rate of any unit of subsystem A/C.

$\mathrm{H}$ : Human-error rate.

$\mu_{K}(s) \Delta:$ The probability that the subsystem $K$ will be repaired in the time interval $(\mathrm{s}, \mathrm{s}+\Delta)$ conditioned that it was not repaired up to time $s$, where $\mathrm{K}=\mathrm{A}, \mathrm{B}, \mathrm{C}$ and $\mathrm{s}=\mathrm{u}, \mathrm{v}, \mathrm{y}, \mathrm{n}, \mathrm{r}$.

$\mu_{h}(m) \Delta \quad: \quad$ The probability that the system will be repaired from state $S_{10}$ in time interval $(\mathrm{m}, \mathrm{m}+\Delta)$ conditioned that it was not repaired up to time $m$.

$S_{i}(x) \quad: \quad \mu_{i}(x) \exp \cdot\left\{-\int_{0}^{\infty} \mu_{i}(x) d x\right\}$

$M_{k} \quad: \quad$ Mean time to repair $\mathrm{k}^{\text {th }}$ unit $=-\bar{S}_{k}{ }^{\prime}(0)$, for all $k$.

$\mathrm{P} \quad$ : Time independent probability.

$D_{k}(s) \quad: \quad\left[1-\bar{S}_{K}(s)\right] / s, \quad$ for all $k=\mathrm{A}, \mathrm{B}$ and C.
A

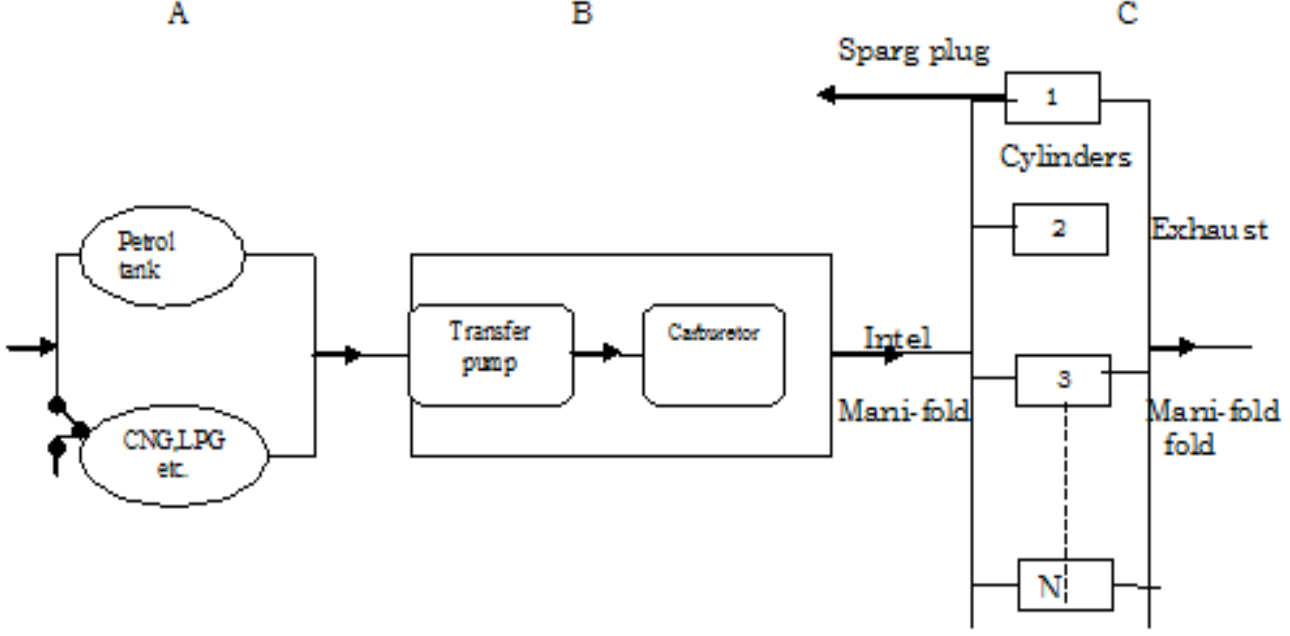

Fig-1: System configuration

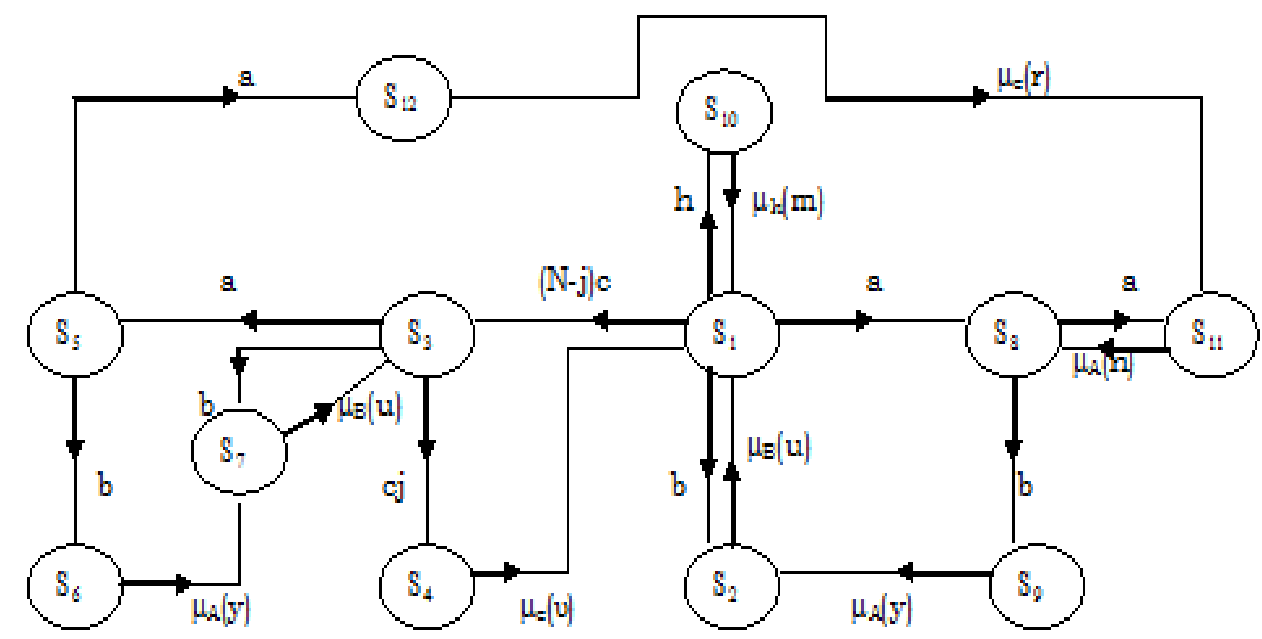

Fig-2: State-transition diagram 


\section{FORMULATION OF THE MATHEMATICAL}

\section{MODEL}

Using continuity argument, we obtain the following set of difference-differential equations governing the behavior of the system under consideration:

$$
\begin{aligned}
& {\left[\frac{d}{d t}+a+b+(N-j) c+h\right] P_{1}(t)=\int_{0}^{\infty} P_{2}(u, t) \mu_{B}(u) d u} \\
& +\int_{0}^{\infty} P_{10}(m, t) \mu_{h}(m) d m+\int_{0}^{\infty} P_{4}(v, t) \mu_{c}(v) d v
\end{aligned}
$$

$\left[\frac{\partial}{\partial u}+\frac{\partial}{\partial t}+\mu_{B}(u)\right] P_{2}(u, t)=0$

$\left[\frac{d}{d t}+c j+b+a\right] P_{3}(t)=(N-j) c P_{1}(t)+\int_{0}^{\infty} P_{7}(u, t) \mu_{B}(u) d u$

$\left[\frac{\partial}{\partial v}+\frac{\partial}{\partial t}+\mu_{c}(v)\right] P_{4}(v, t)=0$

$\left[\frac{d}{d t}+a+b\right] P_{5}(t)=a P_{3}(t)$

$\left[\frac{\partial}{\partial y}+\frac{\partial}{\partial t}+\mu_{A}(y)\right] P_{6}(y, t)=0$

$\left[\frac{\partial}{\partial u}+\frac{\partial}{\partial t}+\mu_{B}(y)\right] P_{7}(u, t)=0$

$\left[\frac{d}{d t}+a+b\right] P_{8}(t)=a P_{1}(t)+\int_{0}^{\infty} P_{11}(n, t) \mu_{A}(n) d n$

$\left[\frac{\partial}{\partial y}+\frac{\partial}{\partial t}+\mu_{A}(y)\right] P_{9}(y, t)=0$

$\left[\frac{\partial}{\partial m}+\frac{\partial}{\partial t}+\mu_{h}(m)\right] P_{10}(m, t)=0$

$\left[\frac{\partial}{\partial n}+\frac{\partial}{\partial t}+\mu_{A}(n)\right] P_{11}(n, t)=0$

$\left[\frac{\partial}{\partial r}+\frac{\partial}{\partial t}+\mu_{c}(r)\right] P_{12}(r, t)=0$

Boundary conditions are: 
$P_{2}(0, t)=b P_{1}(t)+\int_{0}^{\infty} P_{9}(y, t) \mu_{A}(y) d y$

$P_{4}(0, t)=c j P_{3}(t)$

$P_{6}(0, t)=b P_{5}(t)$

$P_{7}(0, t)=b P_{3}(t)$

$P_{9}(0, t)=b P_{8}(t)$

$P_{10}(0, t)=h P_{1}(t)$

$P_{11}(0, t)=a P_{8}(t)+\int_{0}^{\infty} P_{12}(r, t) \mu_{c}(r) d r$

$P_{12}(0, t)=a P_{5}(t)$

Initial conditions are:

$P_{1}(0)=1$, otherwise zero.

\section{SOLUTION OF THE MODEL}

Taking Laplace transforms of equations (1) through (20) by making use of (21) and then on solving them, we have following Laplace transforms of different state probabilities:

$\bar{P}_{1}(s)=\frac{1}{A(s)}$

$\bar{P}_{2}(s)=\frac{b}{A(s)}\left[1+a \bar{S}_{A}(s)\left\{\frac{1}{B(s)}+\frac{a}{s+a+b} \frac{C(s)}{B(s)} \bar{S}_{c}(s) \bar{S}_{A}(s)\right\}\right] D_{B}(s)$

$\bar{P}_{3}(s)=\frac{C(s)}{A(s)}$

$\bar{P}_{4}(s)=c j \frac{C(s)}{A(s)} D_{c}(s)$

$\bar{P}_{5}(s)=\frac{a}{s+a+b} \frac{C(s)}{A(s)}$

$\bar{P}_{6}(s)=\frac{a b}{s+a+b} \frac{C(s)}{A(s)} D_{A}(s)$ 
$\bar{P}_{7}(s)=b \frac{C(s)}{A(s)} D_{B}(s)$
$\bar{P}_{8}(s)=\frac{a}{A(s)}\left[\frac{1}{B(s)}+\frac{a}{s+a+b} \frac{C(s)}{A(s)} \bar{S}_{c}(s) \bar{S}_{A}(s)\right]$

$\bar{P}_{9}(s)=b \bar{P}_{8}(s) D_{A}(s)$

$\bar{P}_{10}(s)=\frac{h}{A(s)} D_{h}(s)$

$\bar{P}_{11}(s)=a\left[\bar{P}_{8}(s)+\frac{a}{s+a+b} \frac{C(s)}{A(s)} \bar{S}_{c}(s)\right] D_{A}(s)$

$\bar{P}_{12}(s)=\frac{a^{2}}{s+a+b} \frac{C(s)}{A(s)} D_{c}(s)$

where, $B(s)=s+b+a\left[1-\bar{S}_{A}(s)\right]$

$C(s)=\frac{(N-j) c}{s+a+c j+b\left[1-\bar{S}_{B}(s)\right]}$

and $\quad A(s)=s+a+b+(N-j) c+h\left[1-\bar{S}_{h}(s)\right]-c j \bar{S}_{c}(s) C(s)-b \bar{S}_{B}(s)$

$-a b \bar{S}_{A}(s) \bar{S}_{B}(s)\left\{\frac{1}{B(s)}+\frac{a}{s+a+b} \frac{C(s)}{B(s)} \bar{S}_{c}(s) \bar{S}_{A}(s)\right\}$

\section{ERGODIC BEHAVIOUR OF THE SYSTEM}

By making use of Abel's Lemma viz., $\lim _{s \rightarrow 0} s \bar{F}(s)=\lim _{t \rightarrow \infty} F(t)=F($ say $)$, provided the limit on

$P_{1}=\frac{1}{A^{\prime}(0)}$

$P_{2}=\frac{1}{A^{\prime}(0)}\left[b+a\left\{1+\frac{a H}{a+b}\right\}\right] M_{B}$

$P_{3}=\frac{H}{A^{\prime}(0)}$

$P_{4}=\frac{c j H}{A^{\prime}(0)} M_{c}$

$P_{5}=\frac{a H}{a+b} \cdot \frac{1}{A^{\prime}(0)}$ right exists, we have the following time independent state probabilities from equations (22) through (33): 
$P_{6}=\frac{a b H}{(a+b) A^{\prime}(0)} M_{A}$

$P_{7}=\frac{b H}{A^{\prime}(0)} M_{B}$

$P_{8}=\frac{a}{b A^{\prime}(0)}\left[1+\frac{a H}{a+b}\right]$

$P_{9}=\frac{a}{A^{\prime}(0)}\left[1+\frac{a H}{a+b}\right] M_{A}$

$P_{10}=\frac{h}{A^{\prime}(0)} M_{h}$

$P_{11}=\frac{a^{2}}{b A^{\prime}(0)}[1+H] M_{A}$

$P_{12}=\frac{a^{2} H}{(a+b) A^{\prime}(0)} M_{c}$

where, $H=\frac{(N-j) c}{a+c j} ;$ and $A^{\prime}(0)=\left[\frac{d}{d s} A(s)\right]_{s=0}$

\section{SOME PARTICULAR CASES}

\subsection{When Repairs Follow Exponential Time Distribution:}

Setting $\bar{S}_{A}(s)=\mu_{A} /\left(s+\mu_{A}\right)$ etc. in equations (22) through (33), we obtained:

$\bar{P}_{1}(s)=\frac{1}{E(s)}$

$\bar{P}_{2}(s)=\frac{b}{E(s)}\left[1+\frac{a \mu_{A}}{s+\mu_{A}}\left\{\frac{1}{F(s)}+\frac{a}{s+a+b} \frac{G(s)}{F(s)} \frac{\mu_{c}}{s+\mu_{c}} \cdot \frac{\mu_{A}}{s+\mu_{A}}\right\}\right] \frac{1}{s+\mu_{B}}$

$\bar{P}_{3}(s)=\frac{G(s)}{E(s)}$

$\bar{P}_{4}(s)=c j \frac{G(s)}{E(s)} \frac{1}{s+\mu_{c}}$

$\bar{P}_{5}(s)=\frac{a}{s+a+b} \frac{G(s)}{E(s)}$

$\bar{P}_{6}(s)=\frac{a b}{s+a+b} \frac{G(s)}{E(s)} \frac{1}{s+\mu_{A}}$ 
$\bar{P}_{7}(s)=b \frac{G(s)}{E(s)} \frac{1}{s+\mu_{B}}$

$\bar{P}_{8}(s)=\frac{a}{E(s)}\left[\frac{1}{F(s)}+\frac{a}{s+a+b} \frac{G(s)}{F(s)} \frac{\mu_{c}}{s+\mu_{c}} \frac{\mu_{A}}{s+\mu_{A}}\right]$

$\bar{P}_{9}(s)=b \bar{P}_{8}(s) \frac{1}{s+\mu_{A}}$

$\bar{P}_{10}(s)=\frac{h}{E(s)} \frac{1}{s+\mu_{h}}$

$\bar{P}_{11}(s)=a\left[\bar{P}_{8}(s)+\frac{a}{s+a+b} \frac{G(s)}{E(s)} \frac{\mu_{c}}{s+\mu_{c}}\right] \frac{1}{s+\mu_{A}}$

$\bar{P}_{12}(s)=\frac{a^{2}}{s+a+b} \frac{G(s)}{E(s)} \frac{1}{s+\mu_{c}}$

where,

$F(s)=s+b+\frac{a s}{s+\mu_{A}} ; \quad G(s)=\frac{(N-j) c}{s+a+c j+\frac{b s}{s+\mu_{B}}}$

and $E(s)=s+a+b+(N-j) c+\frac{h s}{s+\mu_{h}}-c j \frac{\mu_{c}}{s+\mu_{c}} G(s)-\frac{b \mu_{B}}{s+\mu_{B}}$

$-\frac{a b \mu_{A} \mu_{B}}{\left(s+\mu_{A}\right)\left(s+\mu_{B}\right)}\left\{\frac{1}{F(s)}+\frac{a}{s+a+b} \frac{G(s)}{F(s)} \frac{\mu_{c} \mu_{A}}{\left(s+\mu_{c}\right)\left(s+\mu_{A}\right)}\right\}$

\subsection{Availability and Cost Function for the System :}

Now, Laplace transform of availability of petrol engine is given by:

$$
\bar{P}_{u p}(s)=\frac{1}{s+a+b+(N-j) c+h}\left[1+\frac{(N-j) c}{s+a+c j+b}+\frac{a}{s+a+b}\right]
$$

on inverting this, one can obtain the availability at any time ' $t$ ' as under

$$
P_{u p}(t)=Q e^{-[a+b+(N-j) c+h] t}+\operatorname{Re}^{-(a+c j+b) t}+S e^{-(a+b) t}
$$

where $\quad Q=1-\frac{(N-j) c}{(N-2 j) c+h}-\frac{a}{(N-j) c+h}$

$R=\frac{(N-j) c}{(N-2 j) c+h} \quad$ and $\mathrm{S}=\frac{a}{(N-j) c+h}$

Note that $P_{u p}(0)=1$ 
Also, Profit function for the system, working up to the time $t$, is given by

$K(t)=C_{1} \int_{0}^{t} P_{u p}(t) d t-C_{2} t-C_{3}$

where,

$\int_{0}^{t} P_{u p}(t)=Q \frac{1-e^{-(a+b+(N-j) c+h) t}}{a+b+(N-j) c+h}+R \frac{1-e^{-(a+c j+b) t}}{a+c j+b}+S \frac{1-e^{-(a+b) t}}{a+b}$

where, $\mathrm{C}_{1}, \mathrm{C}_{2}$ and $\mathrm{C}_{3}$ are the revenue, repair and establishment cost per unit time respectively also $\mathrm{Q}, \mathrm{R}$ and $\mathrm{S}$ are given in the equations (66)and (67).

\subsection{Steady- State Availability}

The steady state availability of the system is given by

$A_{V S S}=\frac{(a+N c)(a+b)}{b(a+c j)[a+b+(N-j) c+h]}$

Where, $H=(N-j) c / a+c j$

\subsection{Numerical Computation}

For a numerical computation, let us consider the values:

$\mathrm{N}=5, \mathrm{j}=2, \mathrm{a}=0.01, \mathrm{~b}=0.02, \mathrm{c}=0.03, \mathrm{~h}=0.001, \mathrm{C}_{1}=\mathrm{Rs} .5 .00$,

$\mathrm{C}_{2}=\operatorname{Re} 1.00$ and $\mathrm{C}_{3}=\mathrm{Rs} 2.00$.

Also $\mathrm{t}=0,1,2$,

10 and $\mathrm{h}=0.001,0.0015,0.002$

--- 0.01 .

Table-2

\begin{tabular}{|l|l|}
\hline Time & Availability \\
\hline 0 & 1 \\
\hline 1 & 0.976302 \\
\hline 2 & 0.94806 \\
\hline 3 & 0.916399 \\
\hline 4 & 0.882266 \\
\hline 5 & 0.846451 \\
\hline 6 & 0.809611 \\
\hline 7 & 0.772289 \\
\hline 8 & 0.734929 \\
\hline 9 & 0.697892 \\
\hline 10 & 0.661467 \\
\hline 11 & 0.625883 \\
\hline 12 & 0.591317 \\
\hline 13 & 0.557903 \\
\hline 14 & 0.525739 \\
\hline 15 & 0.494891 \\
\hline
\end{tabular}

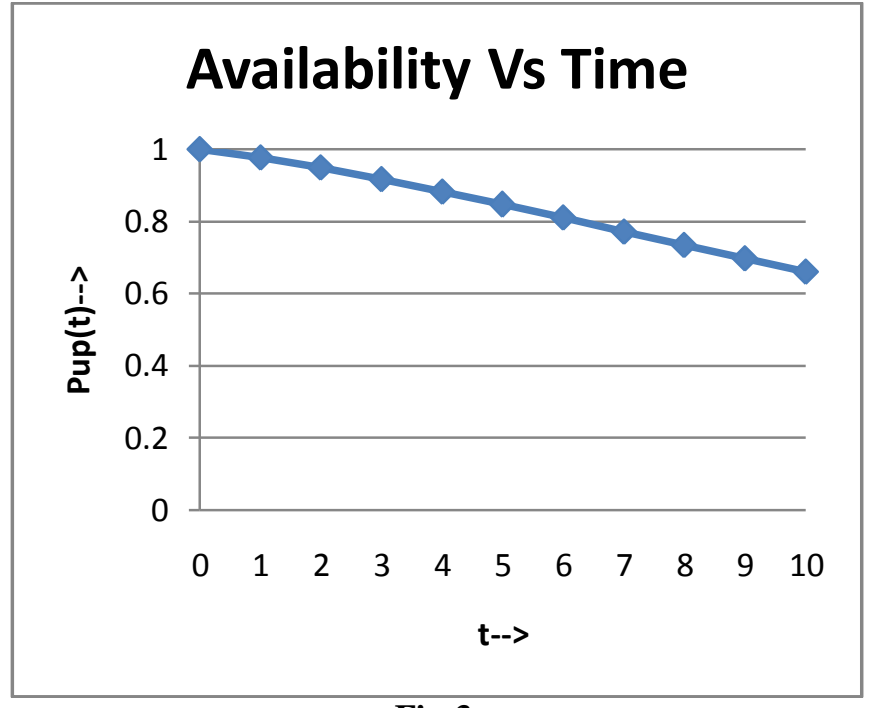

Fig-3

Table-3

\begin{tabular}{|c|c|}
\hline Time & Profit Function \\
\hline 0 & -2 \\
\hline 1 & -0.03425 \\
\hline 2 & 1.853275 \\
\hline 3 & 3.650692 \\
\hline 4 & 5.349202 \\
\hline 5 & 6.942611 \\
\hline 6 & 8.426888 \\
\hline 7 & 9.799798 \\
\hline 8 & 11.06059 \\
\hline 9 & 12.2097 \\
\hline 10 & 13.24855 \\
\hline 11 & 14.17934 \\
\hline 12 & 15.00487 \\
\hline 13 & 15.7284 \\
\hline 14 & 16.35354 \\
\hline 15 & 16.88414 \\
\hline 16 & 17.32424 \\
\hline 17 & 17.67793 \\
\hline 18 & 17.94936 \\
\hline 19 & 18.14267 \\
\hline 20 & 18.26195 \\
\hline
\end{tabular}




\section{Profit Function Vs Time}

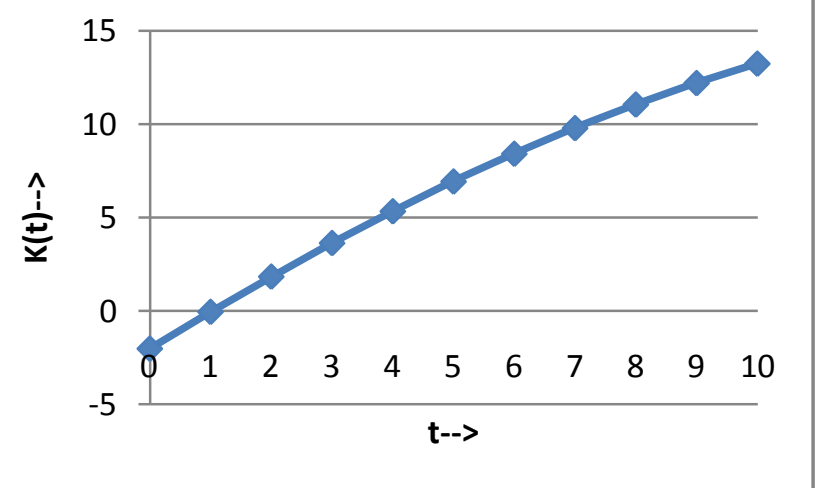

\section{Fig-4}

Table-4

\begin{tabular}{|l|l|}
\hline $\mathbf{h}$ & A VSS \\
\hline 0.001 & 0.283353 \\
\hline 0.0015 & 0.282187 \\
\hline 0.002 & 0.28103 \\
\hline 0.0025 & 0.279883 \\
\hline 0.003 & 0.278746 \\
\hline 0.0035 & 0.277617 \\
\hline 0.004 & 0.276498 \\
\hline 0.0045 & 0.275387 \\
\hline 0.005 & 0.274286 \\
\hline 0.0055 & 0.273193 \\
\hline 0.006 & 0.272109 \\
\hline 0.0065 & 0.271033 \\
\hline 0.007 & 0.269966 \\
\hline 0.0075 & 0.268908 \\
\hline 0.008 & 0.267857 \\
\hline 0.0085 & 0.266815 \\
\hline 0.009 & 0.265781 \\
\hline 0.0095 & 0.264755 \\
\hline 0.01 & 0.263736 \\
\hline
\end{tabular}

\section{Steady- state availability} Vs H

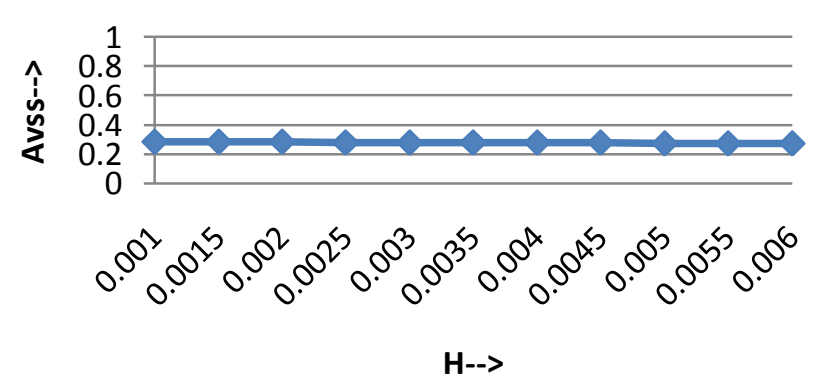

\section{CONCLUSIONS}

The plots of equations (65), (68) and (70) have shown in figs-(3), (4) and (5), respectively.

Fig-3 shows that availability of the system decreases slowly with time initially but there after it decreases approximately in a constant manner.

Fig-4 shows that profit with the system under consideration. For $\mathrm{t}=0$ and 1 , there is no profit but after this profit increases in constant way.

Fig-5 shows long run availability of the system. Its value is 0.283353 . Now as we increase the value of $h$, the long run availability decreases correspondingly.

\section{REFERENCES}

[1] Arora, S.C.; Domkundwar, S.: "A Course in Refrigeration and Air-Conditioning", Dhanpat Rai \& Co. (P) Ltd., 2006.

[2] Billinton, R.; Allan, R.: "Reliability Evaluation of Power Systems", Springer Publication, 1996.

[3] Billinton, R.; Allan, R.: "Reliability Evaluation of Engineering Systems: Concepts and Techniques", Springer Publication, 1992.

[4] Capasso, V.; Bakstein, D.: "An Introduction to Continuous-time Stochastic Processes: Theory, Models and Applications", Birkhauser Publication, 2004.

[5] Culp, W. Archie: "Principles of Energy Conversion", McGraw Hill Book Company, New York, 2000.

[6] Dhillon, B.S.: "Design Reliability", CRC Press, 1999.

[7] Dhillon, B.S.: "Reliability, Quality and Safety Engineers", Taylor Francis, 2004.

[8] Gopal, M.: "Modern Control System Theory", New Age International (P) Ltd., Publishers, India, 2004.

[9] Heyman, D.P.; Sobel, M.J.: "Stochastic Models in Operations Research: Stochastic Processes and Operating Characteristics”, Dover Publication, 2003.

[10] Kuo, W.; Zuo, M.J.: “Optimal Reliability Modeling: Principles and Applications", John Wiley \& Sons., 2002.

[11] Linkov, L.N.: "Asymptotic Statistical Methods for Stochastic Processes", American Mathematical Society, 2001.

[12] Nikolaidis , E.; Ghiocel , D.M. ; Singhal , S. : "Engineering Design Reliability Handbook", CRC Press, 2004.

\section{Research Papers}

[1] Agrafotis, G.S.; Tsoukalas, M. Z.: "Reliability Analysis and Optimization Applications of a Two Unit Standby Redundant System with Spare Units", Microelectronic Reliab. , Vol. 34 (5), pp $1469-$ 1476, 1994. 
[2] Agnihotri, R.R.; Satsangi, S. K.: "Two Non-identical Unit System with Priority Based Repair and Inspection", Microelectronic Reliab. , Vol. 36 (2), pp 279 - 282, 1996.

[3] Cassady, C.R.; Lyoob, I.M.; Schneider, K.; Pohi, E. A.: "A Generic Model of Equipment Availability under Imperfect Maintenance", IEEE TR on Reliability, Vol.54, issue - 4, pp 564 - 571, 2005.

[4] Chung, W.K.: "Reliability Analysis of a Repairable Parallel System with Standby Involving Human Error and Common Cause Failure", Microelectronic Reliab., Vol.27, pp 269-274, 1987.

[5] Chung, W.K.: "Reliability Analysis of a Series System with Repair", Microelectronic Reliab. , Vol.31, pp 363-365, 1991.

[6] Chung, W. K.: "A k-out -of n: G Redundant System with the Presence of Chance with Multiple Critical Errors", Microelectronic Reliab., Vol. 33, pp 334-338, 1993.

[7] Chung, W. K.: " Reliability Analysis of a k-out -of n: G Redundant System in the Presence of Chance with Multiple Critical Errors", Microelectronic Reliab., Vol. 32, pp 331 - 334, 1993.

[8] Cluzeau, T.; Keller, J.; Schneeweiss, W.: “An Efficient Algorithm for Computing the Reliability of Consecutive-k-out-of-n:F Systems", IEEE TR. on Reliability, Vol.57 (1), PP 84-87, 2008.

[9] Dhillon, B. S.; Vishvanath, H. C.: "Reliability Analysis of Non - identical Units Parallel System with Common Cause Failure", Microelectronic Reliab., Vol. 31, pp 429 - 441, 1991.

[10] Dhillon, B. S. and Yang N. J.: "Stochastic Analysis of Standby System with Common Cause Failure and Human Error", Microelectronic Reliab., Vol. 32, pp $1699-1712,1992$. 